Canad. Math. Bull. Vol. 23 (4), 1980

\title{
SOME SPACES ARE NOT THE DOMAIN OF A CLOSED LINEAR OPERATOR IN A BANACH SPACE
}

\author{
PETER DIEROLF AND SUSANNE DIEROLF
}

Let $P(\partial)=\sum_{|\alpha| \leqq m} a_{\alpha} \partial^{\alpha}$ be a linear partial differential operator with $C^{\infty}$ coefficients. The study of $P(\partial)$ as an operator in $L^{2}\left(\mathbb{R}^{n}\right)$ usually starts with the investigation of the minimal operator $P_{0}$ which is the closure of $P(\partial)$ acting on $C_{c}^{\infty}\left(\mathbf{R}^{n}\right)=\mathscr{D}\left(\mathbf{R}^{n}\right)$. In the case of constant coefficients it is known that the domain $D\left(P_{0}\right)$ of $P_{0}$ at least contains the space $\mathscr{S}\left(\mathbf{R}^{n}\right)$ (cf. Schechter [4, p. 58, Lemma 1.2]). However, the question arises whether spaces such as $\mathscr{D}\left(\mathbf{R}^{n}\right)$ or $\mathscr{S}\left(\mathbf{R}^{n}\right)$ may occur as the domain of any closed linear operator in $L^{p}\left(\mathbf{R}^{n}\right)$. The purpose of this note is to show that these and other spaces familiar from the theory of distributions cannot be the domain of a closed linear operator in $L^{p}\left(\mathbf{R}^{n}\right)$. The proofs depend on the following easy consequences of known closed graph theorems.

Proposition. Let $\left(E,\|\cdot\|_{E}\right)$ and $\left(F,\|\cdot\|_{F}\right)$ be Banach spaces. Moreover let $D \subset E$ be a linear subspace and let $A: D \rightarrow F$ be a linear map whose graph $G:=\{(x, A x) \in E \times F ; x \in D\}$ is closed in the product $\left(E,\|\cdot\|_{E}\right) \times\left(F,\|\cdot\|_{F}\right)$. Let $\mathfrak{I}_{1}$ and $\mathfrak{I}_{2}$ denote the topologies on $D$ induced by the norms $\|\cdot\|_{E} \mid D$ and $\|\cdot\|_{A}: D \rightarrow$ $\mathbf{R},\|x\|_{A}:=\|x\|_{E}+\|A x\|_{F}$, respectively.

Clearly $\mathfrak{I}_{2} \supset \mathfrak{I}_{1}$, and $\left(D, \mathfrak{I}_{2}\right)$ is a Banach space. Thus $\operatorname{dim}(D)$ is either finite or uncountable.

(a) $D$ is closed in $\left(E,\|\cdot\|_{E}\right)$ if and only if $\left(D, \mathfrak{T}_{1}\right)$ is barrelled.

(b) Let $\mathfrak{I}$ be a locally convex topology on $D$ which is stronger than $\mathfrak{I}_{1}$. If $(D, \mathfrak{I})$ is barrelled, $\mathfrak{I}$ is stronger than $\mathfrak{I}_{2}$. If $(D, \mathfrak{I})$ is an LF-space (i.e. a countable inductive limit of Fréchet spaces, cf. Grothendieck [2, p. 13]), then $\mathfrak{I}=\mathfrak{I}_{2}$.

Proof. (a) Let $\left(D, \mathfrak{I}_{1}\right)$ be barrelled. Since every Banach space is a Pták space and because of $\mathfrak{I}_{1} \subset \mathfrak{I}_{2}$ the closed graph theorem from Horváth [3, p. 301, Thm. 4] yields $\mathfrak{I}_{1}=\mathfrak{I}_{2}$. Thus $D$ is a complete hence closed subspace of $\left(E,\|\cdot\|_{E}\right)$. The converse statement is trivial.

(b) Because of $\mathfrak{I}_{2} \supset \mathfrak{I}_{1}$ and $\mathfrak{I} \supset \mathfrak{I}_{1}$ the diagonal $\Delta:=\{(x, x) ; x \in D\}$ is a closed subset of $(D, \mathfrak{I}) \times\left(D, \mathfrak{I}_{2}\right)$. If $(D, \mathfrak{I})$ is barrelled, [3, p. 301, Thm. 4] yields that the identity map $(D, \mathfrak{I}) \rightarrow\left(D, \mathfrak{I}_{2}\right)$ is continuous, whence $\mathfrak{I} \supset \mathfrak{I}_{2}$.

Received by the editors April 3, 1979. 
Now let $(D, \mathfrak{I})$ be an $L F$-space. Then $(D, \mathfrak{I})$ is in particular barrelled whence $\mathfrak{I} \supset \mathfrak{I}_{2}$. From [3, p. 304, Prop. 10] we obtain that the identity map $\left(D, \mathfrak{I}_{2}\right) \rightarrow$ $(D, \mathfrak{I})$ is continuous whence $\mathfrak{I}_{2} \supset \mathfrak{I}$.

ExAmples. (1) Let $p \in[1, \infty)$, and let $F$ be a Banach space.

(a) None of the spaces $\mathscr{K}\left(\mathbf{R}^{n}\right), \mathscr{D}^{m}\left(\mathbf{R}^{n}\right)(m \in \mathbf{N}), \mathscr{D}\left(\mathbf{R}^{n}\right)$ (cf. Horváth [3, p. 164 , ex. 5, p. 171 , ex. 8, p. 165 , ex. 6]), and $L_{c}^{p}\left(\mathbf{R}^{n}\right)$ (cf. Treves $[6$, p. 32/33]) can occur as the domain of a closed linear operator from $L^{\mathrm{p}}\left(\mathbf{R}^{n}\right)$ into $F$. In fact, each of these spaces caries a natural $L F$-space-topology, which is stronger than the norm topology induced by $L^{p}\left(\mathbf{R}^{n}\right)$, and which is not normable (Horváth $[3$, p. 175 , ex. 6a]).

(b) None of the spaces $\mathscr{S}\left(\mathbf{R}^{n}\right)$ (Horváth [3, p. 91, ex. 14]) and $\mathscr{D}_{L^{p}}\left(\mathbf{R}^{n}\right)$ (Schwartz [5, p. 199]) can occur as the domain of a closed linear operator from $L^{p}\left(\mathbf{R}^{n}\right)$ into $F$. In fact, these spaces are non-normable Fréchet spaces in their natural topology (thus in particular LF-spaces), and embed continuously into $\mathbf{L}^{p}\left(\mathbf{R}^{n}\right)$.

(2) Let $E, F$ be Banach spaces and let $L$ be a proper dense subspace of $E$ which is barrelled in its relative topology. (On account of Valdivia [7, Thm. 3] $L$ is barrelled if $\operatorname{dim}(E / L)$ is at most countable.) Then there does not exist a linear map $A: L \rightarrow F$ whose graph is closed in $E \times F$.

Now let $(\Omega, \Sigma, \mu)$ be a measure space such that $\operatorname{dim}\left(L^{\infty}(\Omega, \Sigma, \mu)\right)$ is infinite. It follows from the Nikodým boundedness theorem (cf. Diestel, Uhl [1, p. 14, Thm. 1]) that the proper dense subspace $M_{0}(\Sigma):=\operatorname{span}\left\{\chi_{\mathrm{A}} ; A \in \Sigma\right\}$ of $L^{\infty}(\Omega, \Sigma, \mu)$ is barrelled. Thus $M_{0}(\Sigma)$ cannot be the domain of a closed linear operator in $L^{\infty}(\Omega, \Sigma, \mu)$.

(3) Let $I$ be an infinite set and let $p \in[1, \infty) . \mathbf{C}^{(I)}:=\left\{x \in \mathbf{C}^{I} ;\{i \in I ; x(i) \neq 0\}\right.$ is finite $\}$ is not the domain of a closed linear operator in $l^{p}(I)$, since there does not exist a Banach-space-topology on $\mathbf{C}^{(I)}$ stronger than the $\|\cdot\|_{p}$-topology. In fact, $I$ contains a countable infinite subset $J, L:=\left\{x \in \mathbf{C}^{(I)} ; x(i)=0\right.$ for all $\left.i \notin J\right\}$ is a $\|\cdot\|_{p}$-closed linear subspace of $\mathbf{C}^{(I)}$ whose dimension is countably infinite, hence $L$ does not admit a Banach-space-topology.

\section{REFERENCES}

1. J. Diestel and J. J. Uhl, Jr., Vector measures, American Mathematical Society, Providence, Rhode Island 1977.

2. A. Grothendieck, Produits tensoriels topologiques et espaces nucléaires, Memoirs of the AMS, No. 16, American Mathematical Society, Providence, Rhode Island 1966.

3. J. Horváth, Topological vector spaces and distributions, vol. I, Addison-Wesley Publishing Comp., Reading, Massachusetts 1966.

4. M. Schechter, Spectra of partial differential operators, North-Holland Publishing Comp., Amsterdam 1971.

5. L. Schwartz, Théorie des distributions, Hermann, Paris 1966. 
6. F. Treves, Linear partial differential equations with constant coefficients, Gordon and Breach, New York 1966.

7. M. Valdivia, Absolutely convex sets in barrelled spaces, Ann. Inst. Fourier Grenoble 21, 1971, pp. 3-13.

\author{
MATHEMATISCHES INSTITUT \\ DER UNIVERSITÄT MÜNCHEN \\ THERESIENSTR. 39 \\ D-800 München 2 \\ West GeRManY
}

